\title{
Enhanced N-terminal degradation of troponin I blunts cardiac function responsiveness to isoproterenol in 4-week tail-suspended rats
}

\author{
LIN ZHANG, ZHEN SONG, HUI CHANG, YUN-YING WANG and ZHI-BIN YU \\ Department of Aerospace Physiology, Fourth Military Medical University, Xi'an 710032, P.R. China
}

Received June 24, 2012; Accepted October 1, 2012

DOI: $10.3892 / \mathrm{mmr} .2012 .1119$

\begin{abstract}
The N-terminal extension of cardiac troponin I (cTnI) is important in regulating cardiac function. Although the normal rat myocardium shows some cTnI N-terminal degradation (cTnI-ND), exposure to 4 weeks of tail-suspension markedly increased cTnI-ND. We hypothesized that the increased cTnI-ND in tail-suspended rats may affect cardiac function, particularly during $\beta$-adrenergic $(\beta-\mathrm{A})$ stimulation. The increase in cardiac output with isoproterenol (ISO) treatment was smaller in tail-suspended rats compared with controls. Left ventricular end-diastolic pressure was elevated and increases in maximal rates of left ventricular pressure development and relaxation were lower during ISO treatment in tail-suspended rats. Response to ISO, forskolin, DB-cAMP and IBMX was also lower in cardiomyocytes from tailsuspended rats. The increase in shortening and re-lengthening the rates of cardiomyocytes at a maximal dose of ISO, forskolin, DB-cAMP and IBMX treatment was limited in tailsuspended rats. There was no difference in $\mathrm{Ca}^{2+}$ sensitivity of the isometric force between tail-suspended and control rats, although $\mathrm{Ca}^{2+}$ sensitivity was decreased less in tail-suspended rats versus control rats during PKA phosphorylation. There was no difference in PKA protein expression and activation during ISO stimulation between the two groups. Due to the increase in cTnI-ND, ISO-induced phosphorylation of cTnI was reduced in tail-suspended rats. The total phospholamban expression and phosphorylation by ISO was unaltered in tail-suspended rat hearts. These data suggest that enhanced cTnI-ND following 4-week tail-suspension is a major component of the $\beta$-A receptor signaling pathway, depressing cardiac function under ISO stimulation.
\end{abstract}

Correspondence to: Dr. Zhi-Bin Yu, Department of Aerospace Physiology, Fourth Military Medical University, 169 Changlexi Road, Xi'an 710032, P.R. China

E-mail: yuzhib@fmmu.edu.cn

Key words: tail-suspended rat, cardiac function, isoproterenol, cardiac troponin I, degradation

\section{Introduction}

Troponin I (TnI) is a subunit of the troponin complex that regulates the calcium-dependent activation of myofilaments in muscle. Cardiac TnI (cTnI) exhibits a unique N-terminal extension of approximately 30 amino acids. cTnI is not present in the fast skeletal (fsTnI) or slow skeletal (ssTnI) isoforms. The $\mathrm{N}$-terminal extension of cTnI contains two protein kinase A (PKA)-targeted phosphorylation sites [serine (Ser)-23 and Ser-24] (1,2). Phosphorylation of the cTnI N-terminal extension enhances contraction and accelerates relaxation during $\beta$-adrenergic ( $\beta$-A) stimulation by decreased myofibril $\mathrm{Ca}^{2+}$ sensitivity and increased cross-bridge kinetics (3). As such, cTnI is a key regulatory protein in cardiac performance. Hearts of transgenic mice with ssTnI replacing cTnI show reduced relaxation, blunted response to $\beta$-A stimulation (4) and protection against ischemia/reperfusion injury (5), while hearts of transgenic mice with cTnI N-terminal 1-28 amino acid residue deletion show a higher baseline stroke volume and a relaxation rate, similar to wild-type mouse hearts under $\beta$-A stimulation (6). Furthermore, hearts of mice expressing cTnI-S23D/S24D (pseudo-phosphorylation at PKA sites) exhibit constitutive enhancement of rate-dependent increases in systolic and diastolic function in vivo (7). As well as cTnI phosphorylation, the allosteric conformation of cTnI $\mathrm{N}$-terminal extension may be involved in the modulation of cardiac contractile function (8). Transgenic mice with postnatal cardiomyocyte-specific overexpression of a truncated cTnI lacking the acidic $N^{\prime}$ region (cTnI-ND2-11) exhibit significantly reduced rates of cardiac contraction and relaxation under baseline and $\beta$-agonist treatment conditions (9). Overall, these data suggest an important role of $\mathrm{N}$-terminal cTnI phosphorylation in the regulation of cardiac function, particularly on the responsiveness to $\beta$-A stimulation.

The tail-suspended rat is a model used to simulate a cephalic blood shift in microgravity on the ground. The hearts of rats that have been tail-suspended for 4 weeks exhibit increased cTnI degradation, with cTnI cleaved at the 26th, 27th and 30th amino acid residues. Cardiac contractility is also decreased (10). Reduced response to isoproterenol (ISO) in the shortening amplitude and relaxation rate of cardiomyocytes has been demonstrated in 4-week tail-suspended rats (11) and is suggested to be modulated by reduced cAMP (12). However, 
components of cardiac function regulation on the $\beta$-A receptor $(\beta-A R)$ signal transduction pathway are unclear in the tailsuspended rat heart, particularly under $\beta$-A stimulation.

Therefore, the aim of the present study was to test our hypothesis that cTnI N-terminal degradation (cTnI-ND) in 4-week tail-suspended rats is a major component in the reduction of cardiac function responsiveness to $\beta$-A stimulation in the $\beta$-AR signal transduction pathway.

\section{Materials and methods}

Animal model. Two-month-old healthy male Sprague-Dawley rats weighing $220 \pm 10 \mathrm{~g}$ were used. Rats were randomly divided into control (CON) and tail-suspended (SUS) groups. There were 36 rats in each group. All the rats were housed in a $20 \pm 2^{\circ} \mathrm{C}$ environment with a $12: 12 \mathrm{~h}$ light-dark cycle and were fed rat chow and water ad libitum. Tail suspension was performed using the Morey-Holton method for 4 weeks (13). Care was taken to protect the tail tissue and the movement of the rats was not restricted during the procedure. All animal procedures were approved by the Animal Care and Use Committee at the Fourth Military Medical University.

Preparation of isolated working heart. Rats were injected with heparin (100 IU/100 g BW, i.p.) and anesthetized with pentobarbital sodium (40 mg/kg, i.p.). The heart was removed and the aorta was cannulated rapidly. The cannulated hearts were mounted on a heart perfusion apparatus (Radnoti Glass Technology Inc., Monrovia, CA, USA) and perfused with an oxygenated $\left(95 \% \mathrm{O}_{2}-5 \% \mathrm{CO}_{2}\right) \mathrm{Krebs}-H e n s e l e i t$ solution containing (in mM; pH 7.4) $118 \mathrm{NaCl}, 4.7 \mathrm{KCl}, 2.25 \mathrm{MgSO}_{4}$, $2.25 \mathrm{CaCl}_{2}, 23.8 \mathrm{NaCO}_{3}, 1.2 \mathrm{NaH}_{2} \mathrm{PO}_{4}, 0.32$ EDTA and $11.5 \mathrm{D}$-glucose. Following the establishment of coronary perfusion in the Langendorff mode, the left atrium was cannulated through the pulmonary vein with a steel cannula (inner diameter, $1.8 \mathrm{~mm}$; outer diameter, $2.0 \mathrm{~mm}$ ). To detect intraventricular pressure, an ultra-miniature pressure catheter transducer (model SPR-671; Millar Instruments, Houston, TX, USA) was placed into the left ventricle through the left atrium. The preload was set at $10 \mathrm{mmHg}$. The heart was then switched from the Langendorff to the working mode at an afterload of $60 \mathrm{mmHg}$. After the working hearts were equilibrated for $30 \mathrm{~min}$, they were treated with 1,10 or $20 \mathrm{nM}$ ISO (SigmaAldrich, St. Louis, MO, USA) for at least $5 \mathrm{~min}$. Before and after ISO treatment, the aortic flow, coronary flow, heart rate, left ventricular end-systolic (LVESP) and end-diastolic (LVEDP) pressure and maximal rate of left ventricular pressure development $\left(+\mathrm{dP} / \mathrm{dt}_{\max }\right)$ and relaxation $\left(-\mathrm{dP} / \mathrm{dt}_{\max }\right)$ were measured to evaluate cardiac function of the isolated working hearts. Cardiac output is equal to aortic flow plus coronary flow. Data were obtained and analyzed using a PowerLab system and Chart software (ADInstruments Inc., Sydney, Australia).

Cardiomyocyte isolation and unloaded contractile function measurement in the single cardiomyocyte. Single ventricular myocyte isolation was performed as previously described (14). In brief, the cannulated hearts were mounted on a Langendorff perfusion apparatus and perfused with $\mathrm{Ca}^{2+}$-free Joklik's modified minimum essential medium (Sigma-Aldrich) containing $10 \mathrm{mM}$ HEPES and $0.1 \%$ bovine serum albumin (BSA). After
$5 \mathrm{~min}$, the perfusate was switched to a circulating enzyme solution containing $0.08 \%$ collagenase I (Sigma-Aldrich) for $30 \mathrm{~min}$. Perfusion procedures were performed at $37^{\circ} \mathrm{C}$ in a constant flow of $10 \mathrm{ml} / \mathrm{min}$ and the perfusion pressure was monitored. The ventricular tissues were chopped and the cardiomyocytes were dispersed gently by a wide-tipped pipette. The cell suspension was filtered through a $200-\mu \mathrm{m}$ nylon mesh. Cells were resuspended in Joklik's medium containing $1 \% \mathrm{BSA}$ after $30 \mathrm{~min}$ and the $\mathrm{Ca}^{2+}$ concentration gradually recovered to $1.25 \mathrm{mM}$.

Measurements of contractile function were performed in the Edge-Detector system (Crescent Electronics, Sandy, UT, USA) within $6 \mathrm{~h}$ following isolation. Cells were placed into a chamber situated on the stage of an inverted microscope (Olympus IX71; Olympus Co., Ltd., Tokyo, Japan). The cells were perfused with Tyrode's solution containing (in mM; $\mathrm{pH} 7.4$ ) $132 \mathrm{NaCl}, 4.8 \mathrm{KCl}, 1.2 \mathrm{MgCl}_{2}, 1.8 \mathrm{CaCl}_{2}, 5.0$ sodium pyruvate, $10 \mathrm{HEPES}$ and $10 \mathrm{D}$-glucose, at a flow rate of $0.2 \mathrm{ml} / \mathrm{min}$ at $37^{\circ} \mathrm{C}$. Electric field stimulus $(15 \mathrm{~V}, 5 \mathrm{msec}, 2.0 \mathrm{~Hz})$ was administered by the stimulator. The cardiomyocytes were superfused with ISO $(1,5$ and $10 \mathrm{nM})$, forskolin $(0.1,0.5$ and $1.0 \mu \mathrm{M})$, N6,2'-O-dibutyryladenosine 3',5'-cyclic monophosphate sodium (DB-cAMP; 0.1, 0.5 and $1.0 \mathrm{mM}$ ) or 3-isobutyl1-methylxanthine (IBMX; 50, 100 and $200 \mathrm{mM}$ ). Forskolin is an activator of adenylate cyclase. DB-cAMP mimics endogenous cAMP by binding to the PKA regulatory subunit and activates PKA. IBMX is a non-selective phosphodiesterase (PDE) inhibitor and cAMP is degraded by PDEs. Forskolin, DB-cAMP and IBMX were purchased from Sigma-Aldrich.

The shortening amplitude and the maximal rates of shortening $\left(+\mathrm{dL} / \mathrm{dt}_{\max }\right)$ and relaxation $\left(-\mathrm{dL}_{\mathrm{dt}} \mathrm{dt}_{\max }\right)$ were measured by Chart software in the single cardiomyocyte.

Skinned cardiac muscle preparations. Cardiac muscle bundles ( $\sim 0.2 \mathrm{~mm}$ in diameter and 3.6 $\pm 0.4 \mathrm{~mm}$ in length) were dissected from the papillary muscle of the left ventricle under a dissection microscope. Bundles were mounted on a fiber apparatus (Aurora Scientific Inc., Aurora, ON, Canada) for measurement of the isometric force and the sarcomere length (High-speed Video Sarcomere Length Program, Aurora Scientific Inc.). Following equilibration for $30 \mathrm{~min}$, resting force was adjusted to $100 \mathrm{mg}$, sarcomere length was not different at this initial load in any of the muscle bundles $(\mathrm{CON}$, $2.01 \pm 0.04$ vs. SUS, $2.03 \pm 0.07 \mu \mathrm{m})$. Bundles were then chemically skinned with $1 \%$ Triton $\mathrm{X}-100$ for $60 \mathrm{~min}$ in a relaxing solution ( $p \mathrm{Ca}$ 9) containing (in $\mathrm{mM}$; $\mathrm{pH} 7.0$ ) 130 potassium acetate, $1 \mathrm{MgCl}_{2}$, 5 EGTA, $5 \mathrm{Na}_{2} \mathrm{ATP}$, $1 \mathrm{X}$ protease inhibitor cocktail (Roche Diagnostics GmbH, Mannheim, Germany) and 20 imidazole- $\mathrm{HCl}$. A rigor solution (relaxing solution without ATP) was used to examine whether the muscle was completely skinned. Phosphorylation was performed by incubating the muscle bundles with $200 \mathrm{U} / \mathrm{ml}$ catalytic subunit of PKA (Sigma-Aldrich) in relaxing solution for $30 \mathrm{~min}$. The force- $p$ Ca relationship for the cardiac muscle was measured by adding $\mathrm{CaCl}_{2}$ to the relaxing solution to achieve a series of $p \mathrm{Ca}$ (7.0, 6.5, 6.3, 6.0, 5.5, 5.3, 5.0 and 4.5) (15). All experiments were performed at $\sim 22^{\circ} \mathrm{C}$.

Western blot analysis. PKA, TnI and phospholamban (PLB; total and Ser16-phosphorylated) proteins were detected by 
A

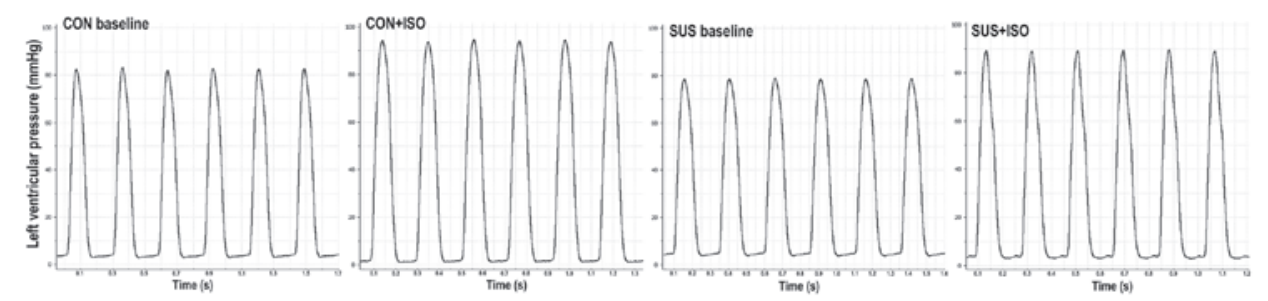

B

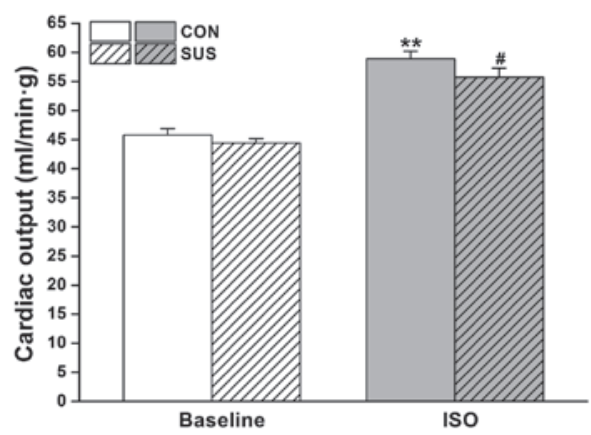

D

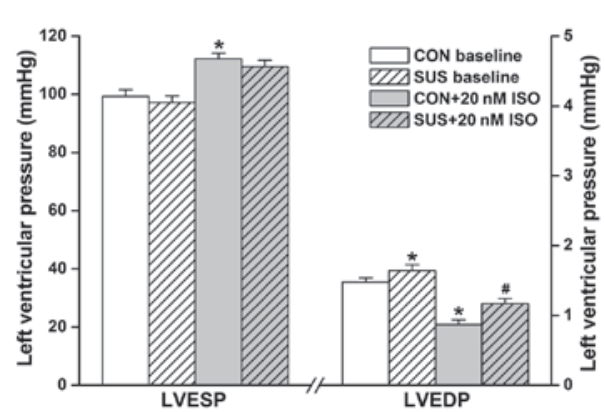

C

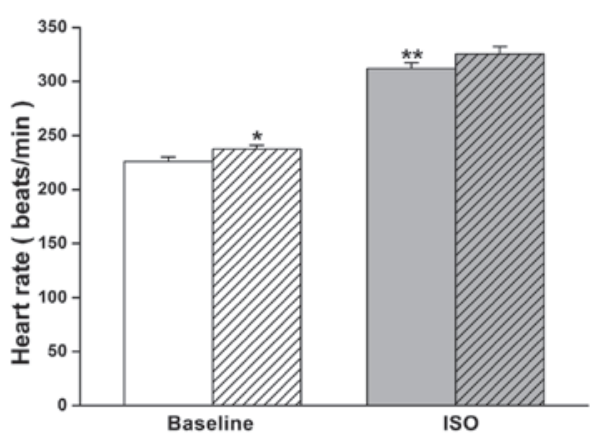

E

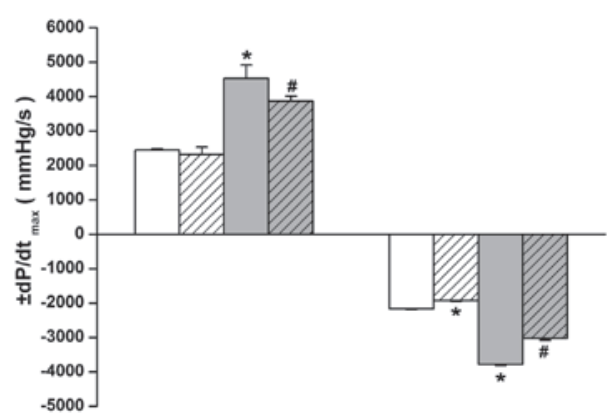

Figure 1. Contractile properties of isolated working hearts before and after ISO stimulation. (A) Representative recordings of the left ventricular pressure. (B) Cardiac output. (C) Heart rate. (D) Left ventricular end-systolic (LVESP) and end-diastolic pressure (LVEDP). (E) Maximal rates of left ventricular pressure development $\left(+\mathrm{dP} / \mathrm{dt}_{\max }\right)$ and relaxation $\left(-\mathrm{dP} / \mathrm{dt}_{\max }\right.$. Data are the mean $\pm \mathrm{SEM} ; \mathrm{n}=6$ hearts/group. ${ }^{*} \mathrm{P}<0.05$ or ${ }^{* * *} \mathrm{P}<0.01$ vs. baseline value of the control group. ${ }^{\#} \mathrm{P}<0.05$ vs. ISO-treated control group. CON, control rats; SUS, tail-suspended rats; ISO, isoproterenol.

western blot analysis. Left ventricular myocardium was homogenized in a buffer containing $50 \mathrm{mM}$ potassium phosphate buffer (pH 7.0), 0.5 mM DTT, $1 \mathrm{mM}$ EDTA, $0.3 \mathrm{mM}$ PMSF and phosphatase inhibitor cocktail (1:100; Sigma-Aldrich). Samples were subjected to SDS-PAGE in polyacrylamide gels (12 or 14\% depending on protein molecular weight). Following electrophoresis, proteins were electrically transferred to nitrocellulose membrane $(0.45 \mu \mathrm{m}$ pore size $)$ using a Bio-Rad semi-dry transfer apparatus (Bio-Rad, Hercules, CA, USA). Blotted nitrocellulose membranes were blocked with $1 \%$ BSA in Tris-buffered saline (TBS; $150 \mathrm{mM} \mathrm{NaCl}, 50 \mathrm{mM}$ Tris- $\mathrm{HCl}$; $\mathrm{pH} 7.5)$ and incubated with rabbit polyclonal anti-PKA C- $\alpha$ [1:10,000; Cell Signaling Technology (CST) Inc., Danvers, MA, USA], mouse monoclonal anti-TnI-I (1:4,000) (10), rabbit polyclonal anti-phosphorylated-Ser23/24 cTnI (1:1,000; CST), rabbit polyclonal anti-desmin (1:1,000; CST), mouse monoclonal anti-PLB $(1: 1,000 ; \mathrm{CST})$ and rabbit polyclonal anti-phosphorylated-Ser16 PLB (Phospho-PLB, 1:1,000; CST) in TBS containing $0.1 \% \mathrm{BSA}$ at $4^{\circ} \mathrm{C}$ overnight. Membranes were incubated in IRDye $680 \mathrm{CW}$ goat anti-mouse or with IRDye $800 \mathrm{CW}$ goat anti-rabbit secondary antibodies $(1: 10,000)$ for $90 \mathrm{~min}$ at room temperature (RT) and visualized using an Odyssey scanner (LI-COR Biosciences, Lincoln,
NE, USA). Quantification analysis of blots was performed with the NIH Image J software (available at http://rsbweb.nih. gov/ij/download.html).

Immunofluorescent histochemistry and confocal analysis. Cardiomyocytes were incubated in a $5 \% \mathrm{CO}_{2}$ incubator at $37^{\circ} \mathrm{C}$ until cells attached to the dish coated with $10 \mu \mathrm{g} / \mathrm{ml}$ laminin (Sigma-Aldrich). The cells were exposed to $10 \mathrm{nM} \mathrm{ISO}$ for $60 \mathrm{~min}$ and then fixed in $4 \%$ paraformaldehyde for $30 \mathrm{~min}$. The cells were permeabilized in $0.1 \%$ Triton X-100/PBS for 30 min, blocked with $1 \%$ BSA in PBS for $60 \mathrm{~min}$ at RT and then incubated with rabbit polyclonal anti-PKA C- $\alpha$ antibody (1:100) at $4^{\circ} \mathrm{C}$ overnight. The slides were rinsed twice in PBS and incubated with tetramethylrhodamine (TRITC)-labeled goat anti-rabbit IgG (1:400; Molecular Probes, Eugene, OR, USA) for $60 \mathrm{~min}$. The slides were then washed in PBS, incubated in Hoechst $33258(5 \mu \mathrm{g} / \mathrm{ml})$ for $30 \mathrm{~min}$ and washed twice with PBS. Staining was observed using a laser-scanning confocal microscope (Olympus FV1000; Olympus Co., Ltd.) equipped with the FV10-ASW system. TRITC- and Hoechst-labeled signals were visualized at 555 and $352 \mathrm{~nm}$, respectively. Images were captured at x60 water objective. Image optical densitometry analysis was performed using 
A
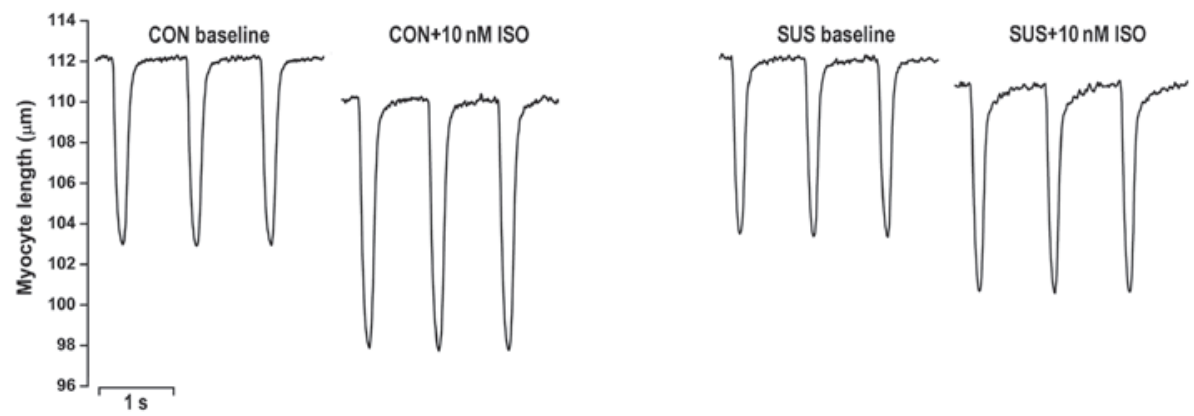

B

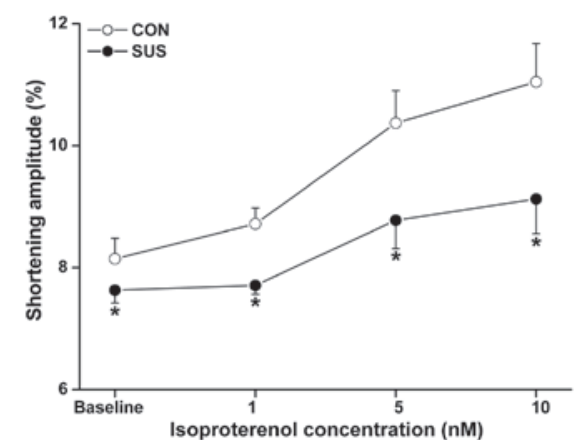

D

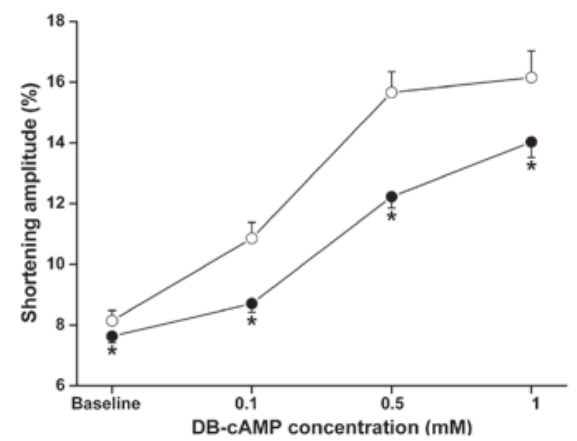

C

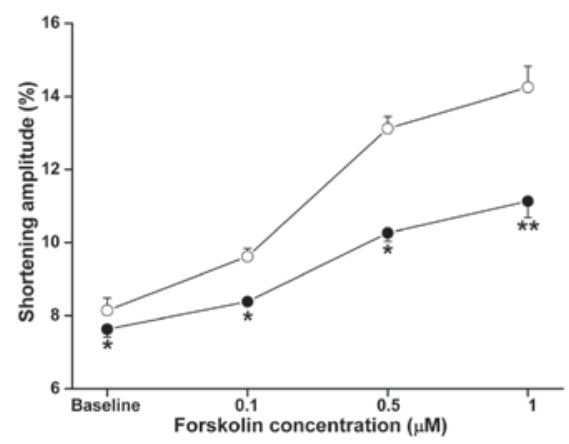

$\mathbf{E}$

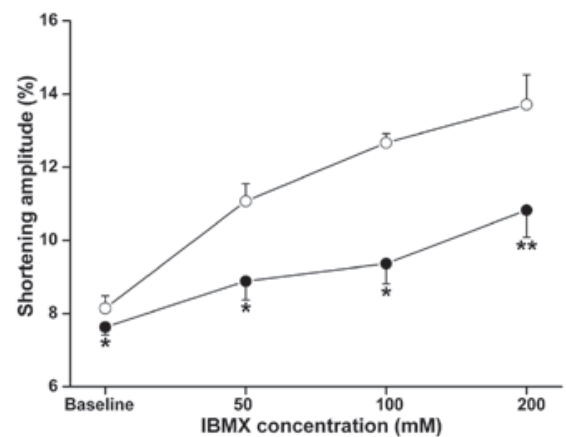

Figure 2. Unloaded contractile functions of cardiomyocytes with ISO, forskolin, DB-cAMP and IBMX treatments. Cardiomyocytes were stimulated at 2.0 Hz. The shortening amplitude of cardiomyocytes was measured in each group. (A) Representative recordings of unloaded contraction of single cardiomyocytes. (B) ISO perfusion at 1,5 and $10 \mathrm{nM}$. (C) Forskolin perfusion at $0.1,0.5$ and $1.0 \mu \mathrm{M}$. (D) DB-cAMP perfusion at 0.1, 0.5 and $1.0 \mathrm{mM}$. (E) IBMX perfusion at 50, 100 and $200 \mathrm{mM}$. Data are the mean $\pm \mathrm{SEM} ; \mathrm{n}=18$ myocytes from six hearts for each agonist in each group. ${ }^{*} \mathrm{P}<0.05$ or ${ }^{* *} \mathrm{P}<0.01$ vs. the control group. CON, control rats; SUS, tail-suspended rats; ISO, isoproterenol; DB-cAMP, N6,2'-O-dibutyryladenosine 3',5'-cyclic monophosphate sodium; IBMX, 3-isobutyl-1-methylxanthine.

Olympus Fluoview image analysis software (Olympus Co., Ltd.).

Statistical analysis. Data were presented as the mean \pm SEM. Differences between the two groups were compared by the unpaired Student's t-test. For multi-group comparisons, two-way ANOVA followed by Tukey post-hoc test was performed. $\mathrm{P}<0.05$ was considered to indicate a statistically significant difference.

\section{Results}

Reduced responsiveness of isolated working heart to ISO in tail-suspended rats. Body weights of tail-suspended rats were similar to those of age-matched controls (CON, 304.7 $\pm 11.9 \mathrm{~g}$ vs. SUS, $287.5 \pm 11.9 \mathrm{~g} ; \mathrm{P}>0.05$ ). The wet weight of the hearts in the tail-suspended rats was also not found to be significantly different from those in the control rats $(\mathrm{CON}, 930.2 \pm 37.5 \mathrm{mg}$ vs. SUS, 931.9 $\pm 24.3 \mathrm{mg} ; \mathrm{P}>0.05$ ).

Cardiac function in working heart mode was assessed at a preload of $10 \mathrm{mmHg}$ and an afterload of $60 \mathrm{mmHg}$. Basal cardiac output values were similar between control and tail-suspended rats $(\mathrm{P}>0.05)$. ISO stimulation increased cardiac output in the control and tail-suspended groups, but there was a higher increase in cardiac output following $20 \mathrm{nM}$ ISO treatment in the control compared with the tail-suspended group (Fig. 1B). The intrinsic the heart rate was $225.9 \pm 4.1$ beats $/ \mathrm{min}$ in the control group and $237.5 \pm 3.5$ beats $/ \mathrm{min}$ in the tail-suspended group. During $20 \mathrm{nM}$ ISO perfusion, heart rate increased to $312 \pm 5.4$ beats $/ \mathrm{min}$ in the control and to $325.5 \pm 6.7$ beats $/ \mathrm{min}$ in the tail-suspended group. The basal heart rate was found to be significantly higher in the tail-suspended group compared with the control group (Fig. 1C).

ISO treatment induced a significant increase in LVESP of the control $(\mathrm{P}<0.05)$ and the tail-suspended group $(\mathrm{P}<0.05)$, while no difference in LVESP between the tail-suspended and control groups with or without ISO treatment was observed (Fig. 1D). Basal LVEDP was higher in the tail-suspended group than the control group $(\mathrm{P}<0.05)$. A significant decrease in LVEDP following perfusion with $20 \mathrm{nM}$ ISO was found in the groups, which was more marked in the control than in the tail-suspended group ( $\mathrm{P}<0.05$, Fig. 1D). 


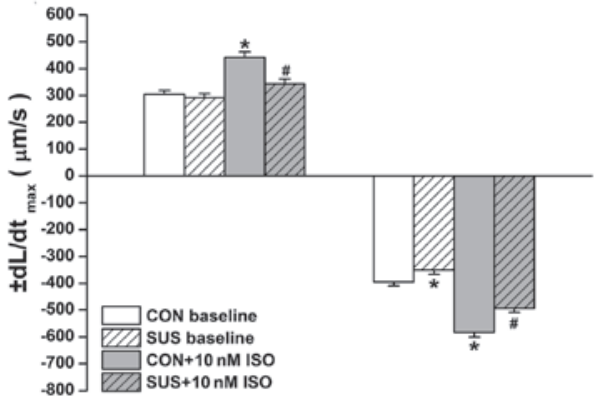

B

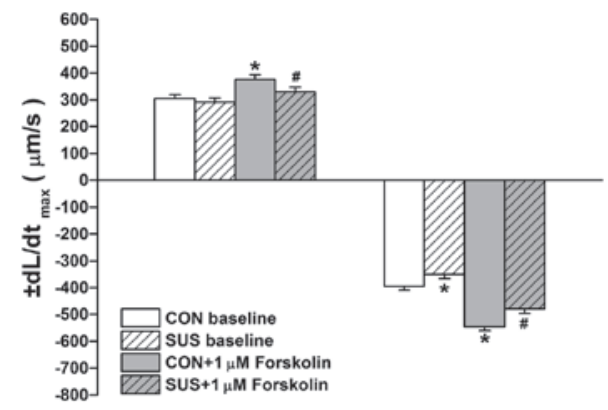

C

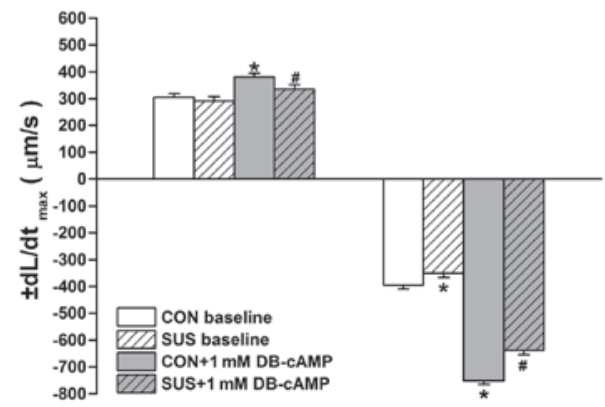

D

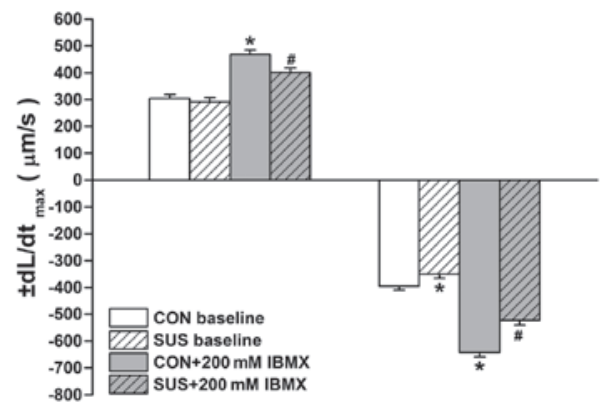

Figure 3. Effects of ISO, forskolin, DB-cAMP and IBMX on maximal rates of shortening $\left(+\mathrm{dL}^{-} \mathrm{dt}_{\max }\right)$ and relaxation $\left(-\mathrm{dL} / \mathrm{dt}_{\max }\right)$ in cardiomyocytes. Treatment of (A) $10 \mathrm{nM}$ ISO, (B) $1.0 \mu \mathrm{M}$ forskolin, (C) $1 \mathrm{mM}$ DB-cAMP, (D) $200 \mathrm{mM}$ IBMX is shown. Data are the mean $\pm \mathrm{SEM}$; $\mathrm{n}=18$ myocytes from 6 hearts for each agonist in each group. ${ }^{*} \mathrm{P}<0.05$ vs. baseline value of the control group. ${ }^{~} \mathrm{P}<0.05$ vs. ISO-treated control group. CON, control rats; SUS, tailsuspended rats; ISO, isoproterenol; DB-cAMP, N6,2'-O-dibutyryladenosine 3',5'-cyclic monophosphate sodium; IBMX, 3-isobutyl-1-methylxanthine.

Under baseline conditions, no change in $+\mathrm{dP} / \mathrm{dt}_{\max }$ was observed in the tail-suspended group, while $-\mathrm{dP} / \mathrm{dt}_{\max }$ was found to be significantly reduced when compared with the control group $(\mathrm{P}<0.05$; Fig. 1E). Following $20 \mathrm{nM}$ ISO treatment, a significant increase in $+\mathrm{dP} / \mathrm{dt}_{\max }$ and $-\mathrm{dP} / \mathrm{dt}_{\max }$ was found in the control $(\mathrm{P}<0.05)$ and tail-suspended groups $(\mathrm{P}<0.05)$ compared with the values prior to ISO administration.
Increase in $\pm \mathrm{dP} / \mathrm{dt}_{\max }$ in response to ISO was greater in the control group than the tail-suspended group $(\mathrm{P}<0.05$, Fig. 1E).

Depressed responsiveness of cardiomyocytes to $\beta$-AR signaling pathway agonists in tail-suspended rats. To determine the major mediator(s) regulating the ISO responsiveness in tail-suspended rats, we examined the effects of the upstream agonists of the $\beta$-AR signaling pathway in cardiomyocytes at a stimulation frequency of $2.0 \mathrm{~Hz}$. The baseline value in shortening amplitude of cardiomyocytes was lower in the tailsuspended group than in the control group (Fig. 2). Exposure to ISO, forskolin, DB-cAMP and IBMX produced dose-dependent positive inotropic responses in control and tail-suspended rat cardiomyocytes. These responses were significantly lower in the tail-suspended group than in the control group (Fig. 2).

The increase in cardiomyocyte shortening amplitude following ISO stimulation (1,5 and $10 \mathrm{nM}$ ) was less marked in tail-suspended rats compared with control rats (Fig. 2B). Forskolin $(0.1,0.5$ and $1.0 \mu \mathrm{M})$, an activator of adenylate cyclase, induced a smaller increase in shortening amplitude in the tail-suspended group (Fig. 2C). DB-cAMP is a cell membrane-permeable and PDE-resistant cAMP analog. Exposure to 0.1, 0.5 and 1.0 mM DB-cAMP induced a smaller increase in cardiomyocyte shortening amplitude in tailsuspended rats compared with the control (Fig. 2D). IBMX (50, 100 and $200 \mathrm{mM}$ ), a non-selective inhibitor of PDEs, also induced a smaller increase in shortening amplitude in the tailsuspended group (Fig. 2E).

Baseline values of $+\mathrm{dL} / \mathrm{dt}_{\max }$ of cardiomyocytes were similar between the control and tail-suspended groups, while the baseline values of $-\mathrm{dL} / \mathrm{dt}_{\max }$ decreased significantly in the tail-suspended compared with the control group $(\mathrm{P}<0.05$; Fig. 3A). ISO, forskolin, DB-cAMP and IBMX all enhanced the $+\mathrm{dL} / \mathrm{dt}_{\max }$ and $-\mathrm{dL}_{\mathrm{dt}} \mathrm{t}_{\max }$ of cardiomyocytes in the control and tail-suspended groups $(\mathrm{P}<0.05$; Fig. 3$)$. The increased $+\mathrm{dL}^{-\mathrm{dt}_{\max }}$ and $-\mathrm{dL}^{\mathrm{d} t_{\max }}$ in response to ISO, forskolin, DB-cAMP or IBMX treatments were significantly less in the tail-suspended group than the control ( $\mathrm{P}<0.05$; Fig. 3).

$P K A$ reduced myofibrillar $\mathrm{Ca}^{2+}$ sensitivity in the myocardium. The maximum $\mathrm{Ca}^{2+}$-activated isometric force of skinned muscle fibers from control rats was significantly higher than that from tail-suspended rats (10). No difference was observed in the myocardial isometric force- $p \mathrm{Ca}$ relationship between the two groups (Fig. 4). Although PKA induced a reduction in myofibrillar $\mathrm{Ca}^{2+}$ sensitivity in the control and tail-suspended groups, the reduction was less marked in tail-suspended rats. The $\left[\mathrm{Ca}^{2+}\right]$ required for $50 \%$ activation $(p \mathrm{Ca} 50)$ was 6.07 $\pm 0.17 p$ Ca units at baseline and 5.93 \pm 0.09 following PKA treatment in tail-suspended rat myofibrils, compared with $6.08 \pm 0.12 p \mathrm{Ca}$ units at baseline and 5.79 \pm 0.11 following PKA treatment in the control rats $(\mathrm{P}<0.05)$.

Increased $N$-terminal degradation of cTnI and reduced cTnI phosphorylation by ISO in the tail-suspended group. Western blot analysis revealed two bands of cTnI in hearts from the control and tail-suspended rats (Fig. 5A). The percentage of intact cTnI (upper band)/total cTnI (intact cTnI plus cTnI fragment) was decreased in tail-suspended rat hearts compared with the control $(\mathrm{P}<0.05)$. Optical densitometry of the cTnI 


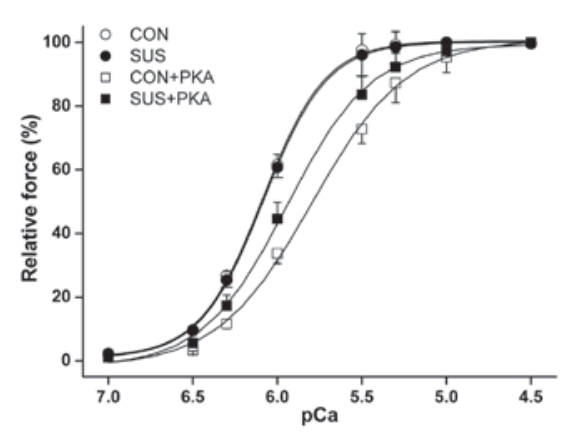

Figure 4. Effects of phosphorylation by PKA on the force- $p$ Ca relationships of skinned cardiac muscles from control and tail-suspended rats. Force is expressed relative to the maximum force before PKA. Lines with the least squares fits to the Hill equation. Data are the mean \pm SEM; $n=6$ muscle bundles from 6 hearts/group. CON, control rats; SUS, tail-suspended rats; PKA, protein kinase A.

fragment (lower band) was $15.5 \pm 1.3 \%$ of total $\mathrm{cTnI}$ in the control and $23.0 \pm 0.7 \%$ in the tail-suspended group (Fig. 5B), with significantly greater cTnI degradation in the tailsuspended group versus the control group $(\mathrm{P}<0.05)$. The basic phosphorylation level of cTnI was significantly decreased in the tail-suspended group compared with the control $(\mathrm{P}<0.05$; Fig. 5A and C). Following ISO stimulation, phosphorylated cTnI increased in tail-suspended and control groups, however, the increase was smaller in the tail-suspended group than that in the control $(\mathrm{P}<0.05 ;$ Fig. 5C).

Expression and activation of PKA were unaltered in the tailsuspended group. Inactive PKA is a heterotetramer composed of a regulatory subunit (R) dimer and a catalytic subunit (C) dimer. In its inactive state, pseudosubstrate sequences on the $\mathrm{R}$ subunits block the active sites on the $\mathrm{C}$ subunits. When cAMP binds to the $\mathrm{R}$ subunits, the auto-inhibitory contact is relieved and active monomeric $\mathrm{C}$ subunits are released. Three $\mathrm{C}$ subunit isoforms $(\mathrm{C}-\alpha,-\beta$ and $-\gamma)$ have been identified and $\mathrm{C}-\alpha$ is predominantly expressed in the heart. Thus, using PKA C- $\alpha$ subunit antibody we detected activated PKA. Compared with desmin, no difference in the expression of the activated PKA C- $\alpha$ subunit between the control and tailsuspended groups before and after $10 \mathrm{nM}$ ISO stimulation was observed (Fig. 6A and B). Immunofluorescence data revealed a similar degree of PKA translocation into the sarcolemma of cardiomyocytes following $10 \mathrm{nM}$ ISO stimulation between the control and tail-suspended groups (Fig. 6C and D).

Expression and Serl6 phosphorylation of PLB were unchanged in the tail-suspended group. The level of total PLB and/or phosphorylation at Ser16 (the residue phosphorylated by PKA) are important factors that may alter relaxation function in tail-suspended rat hearts. However, there was no change in the expression or phosphorylation of PLB at Ser16 by western blot analysis following ISO stimulation (Fig. 7).

\section{Discussion}

In the present study, the baseline LVEDP of the working heart was higher in the tail-suspended rats compared with the control, while a blunted response to ISO in all parameters
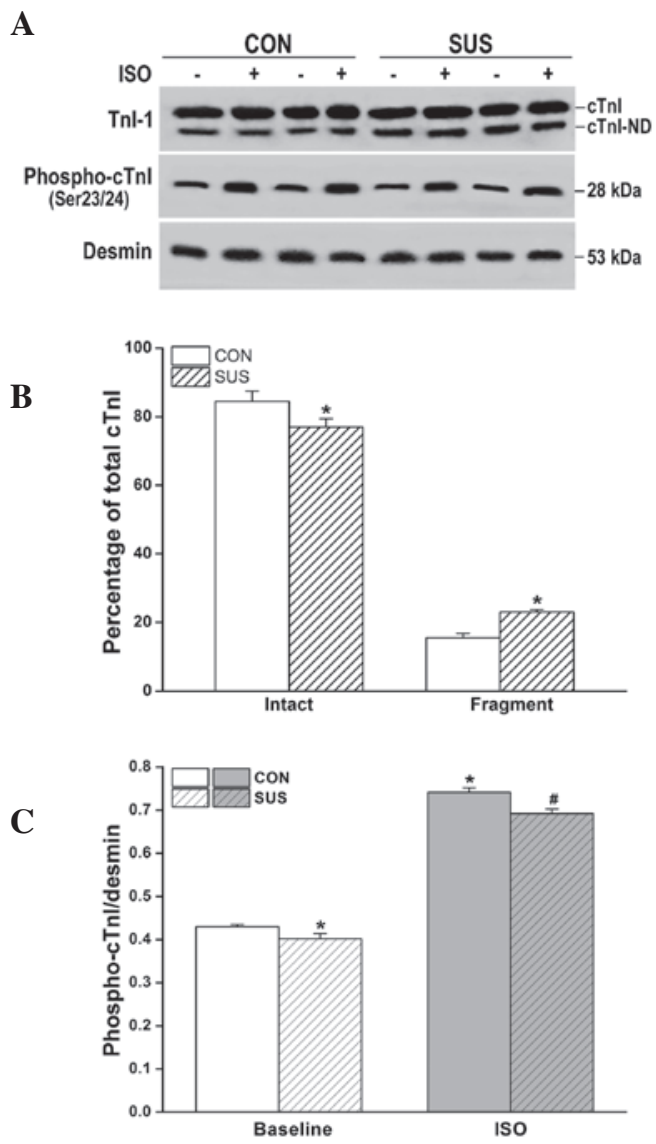

Figure 5. N-terminal degradation and phosphorylation of cTnI in control and tail-suspended rats. (A) Representative immunoblots and mean data showing (B) N-terminal degradation and (C) phosphorylation of $\mathrm{cTnI}$ ( $\mathrm{n}=6$ hearts per group). Data are the mean \pm SEM. ${ }^{*} \mathrm{P}<0.05$ vs. baseline value of the control group. ${ }^{\#} \mathrm{P}<0.05$ vs. ISO-treated control group. CON, control rats; SUS, tail-suspended rats; TnI-1, troponin I 1; cTnI-1, cardiac TnI-1.

measured in the working heart, isolated cardiomyocytes and skinned fibers was identified in tail-suspended rats. Thus, the decrease in ISO sensitivity with tail-suspension was associated with changes in the $\beta$-AR signaling pathway at the level of the individual cardiomyocyte.

Stimulation of $\beta$-AR by ISO activates $\mathrm{G}_{\mathrm{s}}$, which in turn activates adenylate cyclase and increases the formation of cAMP within cardiomyocytes. Elevated levels of cAMP increase activation of PKA, which phosphorylates intracellular targets, including L-type $\mathrm{Ca}^{2+}$ channels, ryanodine receptor (RyR), PLB, cTnI and myosin-binding protein C (MyBPC). Therefore, the upstream components in the $\beta$-AR signal transduction pathway include $\beta-A R, G_{s}$, adenylate cyclase, cAMP and PKA. The downstream components are the targeting proteins of PKA.

Forskolin, a direct adenylate cyclase agonist, caused a smaller increase in the shortening amplitude and the maximal rate of relaxation in tail-suspended rat cardiomyocytes. By contrast, a reduction in cAMP of cardiomyocytes has been reported to depress the response to ISO in tail-suspended rats (12). However, in the present study, response to DB-cAMP was less in the cardiomyocytes of tail-suspended rats compared with the control. In addition, under basal conditions and during $\beta$-AR stimulation, intracellular cAMP levels are regulated by PDE, which catalyzes the breakdown 


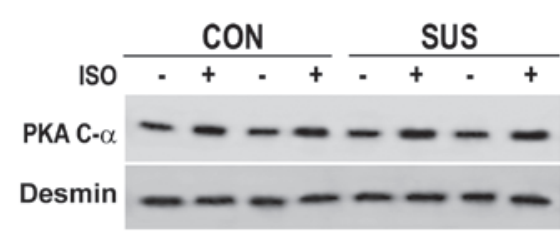

B

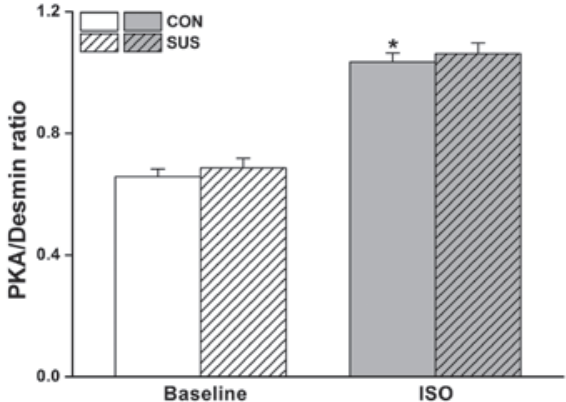

$\mathbf{C}$

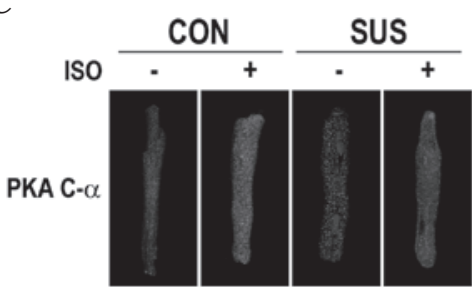

D

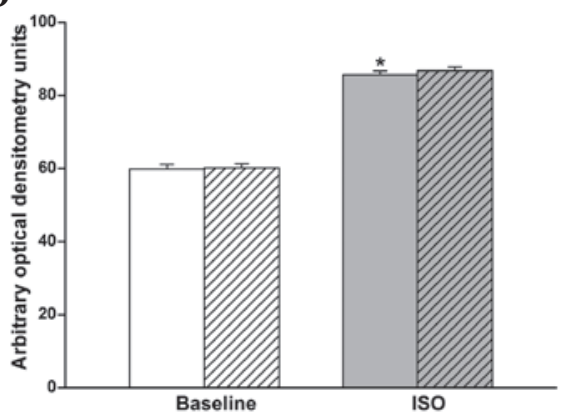

Figure 6. Expression and translocation of PKA before and after $10 \mathrm{nM}$ ISO stimulation in the control and tail-suspended rats. (A and B) Representative immunoblots and mean data showing activated PKA before and after ISO stimulation. Data are the mean \pm SEM; $n=6$ hearts per group. (C) Representative immunofluorescence of cardiomyocytes for PKA with tetramethylrhodamine, scanned at the cell membrane level. (D) Mean data showing the translocation of PKA to the membrane after ISO treatment. Data are the mean \pm SEM; $n=9$ myocytes from 3 hearts/group. ${ }^{*} \mathrm{P}<0.05$ vs. baseline value of the control group. CON, control rats; SUS, tail-suspended rats; ISO, isoproterenol; PKA, protein kinase A.

A

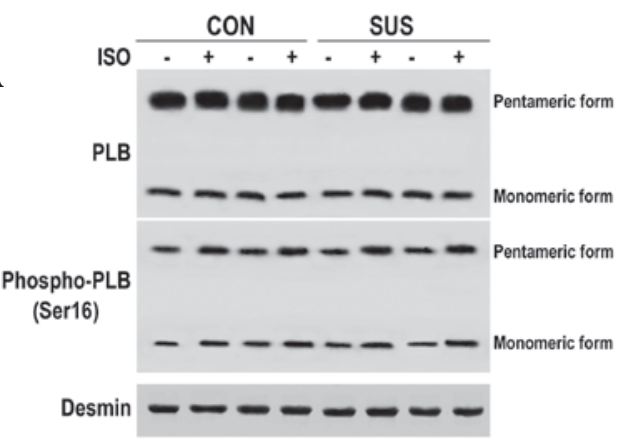

B

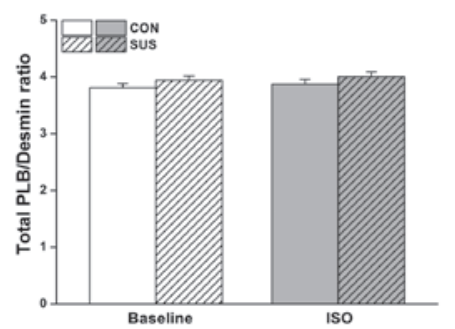

C

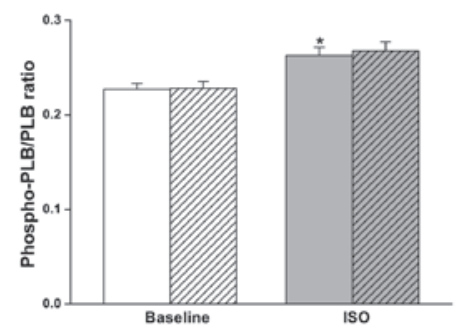

Figure 7. Expression of PLB and phospho-PLB at Ser16 before and after 10 nM ISO treatment in the control and tail-suspended rats. (A) Representative immunoblots and mean data showing the (B) expression of PLB and (C) phospho-PLB (Ser16). PLB was detected in its pentameric and monomeric forms. Data are the mean \pm SEM; $n=6$ hearts/group. ${ }^{*} \mathrm{P}<0.05$ vs. baseline value of the control group. CON, control rats; SUS, tail-suspended rats; ISO, isoproterenol; PLB, phospholamban; phospho-PLB, phosphorylated PLB. of cAMP. The PDE inhibitor IBMX did not reverse the tailsuspension-related deficit in positive inotropy caused by $\beta$-AR stimulation. PKA expression and membrane translocation of cardiomyocytes during $\beta$-AR stimulation was similar in both the tail-suspended and control rats. These findings suggest that events downstream of PKA in the $\beta$-AR signal transduction pathway may be affected by the tail-suspension.

Phosphorylation of L-type $\mathrm{Ca}^{2+}$ channels enhances $\mathrm{Ca}^{2+}$ influx, increasing the shortening amplitude or force of contraction, but does not regulate the relaxation of cardiomyocytes. A previous study demonstrated that adrenergic regulation of cardiac contractility does not involve phosphorylation of the cardiac ryanodine receptor at Ser2808 by PKA (16). Phosphorylation of MyBPC does not appear to have any effect on myofibrillar $\mathrm{Ca}^{2+}$ sensitivity of the isometric force, which modulates the relaxation, but accelerates kinetics of force development (17). Phosphorylated MyBPC is only involved in myocardial protection during ischemia $(18,19)$. This indicates that phosphorylation of PLB and CTnI is important in the regulation of cardiac function, particularly the relaxation function of cardiomyocytes downstream of the $\beta$-AR signal transduction pathway.

Phosphorylation of PLB ameliorates the inhibition of SERCA and increases $\mathrm{Ca}^{2+}$ uptake by SERCA. Thus, the rate of relaxation is enhanced during $\beta$-AR stimulation due to increased sequestration of $\mathrm{Ca}^{2+}$ by increased SERCA activity. In PLB knockout mice, phosphorylation of PLB has been shown to account for approximately $50 \%$ of the enhanced relaxation rate effect (20). However, there was no difference in total PLB and PLB phosphorylation at the PKA-targeted site Ser16 residue in cardiomyocytes between the tail-suspended and control rats. cTnI, another substrate phosphorylated by PKA, exhibited N-terminal degradation in cardiomyocytes of control and tail-suspended rats, although the $\mathrm{N}$-terminal degradation of cTnI was more marked in tail-suspended rats. In turn, 
the increased $\mathrm{N}$-terminal degradation of $\mathrm{cTnI}$ reduced phosphorylation in tail-suspended rats. Thus, these data strongly suggest that the blunted cardiac function in $\beta$-AR stimulation is correlated with enhanced cTnI N-terminal degradation in the tail-suspended rats.

A number of mechanistic systems models have been developed to analyze the functional roles of PLB, L-type calcium channel, RyR and cTnI phosphorylation upon $\beta$-AR stimulation in rat ventricular myocytes. The model analysis revealed that the PKA-mediated phosphorylation of cTnI only exhibits a nominal lusitropic response during $\beta$-AR stimulation (21). However, transgenic mice expressing ssTnI specifically in cardiomyocytes exhibit less shortening and prolongation of the half-time of intracellular $\left[\mathrm{Ca}^{2+}\right]$ decay, while similar transgenic cardiomyocytes show no enhancement of the velocity of shortening during isoprenaline treatment (4). By using PLB knockout transgenic mice, cTnI phosphorylation has been shown to contribute $14-18 \%$ of the lusitropic effect during maximal isometric contractions (20). Overall, these data indicate that phosphorylation of cTnI at the N-terminus by PKA is a key modulator of cardiac function. In addition, the allosteric conformation of cTnI N-terminal extension also regulates cardiac contractile function (8). Degradation of $\mathrm{N}$-terminal extension induces a change in the allosteric conformation of cTnI. Sadayappan et al (9) have generated transgenic mice with a truncated cTnI that lacks the acidic $\mathrm{N}^{\prime}$ region (cTnI-ND2-11). The acidic $\mathrm{N}^{\prime}$ region is not involved in the phosphorylated sites of cTnI, although cTnI-ND2-11 hearts exhibit significantly reduced rates of contraction and relaxation under baseline and $\beta$-agonist treatment (9).

cTnI has been previously shown to be cleaved at the 26th, 27th and 30th amino acid residues in tail-suspended rat hearts (10). N-terminal degradation of cTnI decreased the degree of total cTnI phosphorylation by PKA in the hearts of tail-suspended rats. Although cTnI-ND accounted for only approximately $20 \%$ of total cTnI in tail-suspended rats in the present study, this cTnI-ND lacked the PKA phosphorylation sites, Ser23 and Ser24, changing the allosteric conformation of cTnI N-terminal extension. These combined effects may enhance the modulation of the cardiac function in tail-suspended rat hearts. Therefore, in the present study, increased cTnI-ND reduced the contraction and relaxation function of the left ventricle in working hearts and impaired cardiac function of cardiomyocytes in tail-suspended rats at baseline and during $\beta$-AR stimulation. Barbato et al reported that the transgenic mouse hearts with the N-terminal truncation of cTnI reduced LVEDP at the basic level, but the response of $\pm \mathrm{dP} / \mathrm{dt}_{\max }$ to ISO was also lower in transgenic mice than wild-type mice (6).

Furthermore, cTnI phosphorylation at Ser23 and Ser24 by PKA has been shown to depress the $\mathrm{Ca}^{2+}$ affinity and $\mathrm{Ca}^{2+}$ off-rate of cTnC in vitro (22). A rapid dissociation of $\mathrm{Ca}^{2+}$ from cTnC is another important factor that accelerates the relaxation rate during $\beta$-agonist treatment. Removal of the N-terminal domain of cTnI from 1-28 amino acid residues has been shown to decrease the $\mathrm{Ca}^{2+}$ sensitivity of actomyosin ATPase in the transgenic mouse myocardium (6). The lower $\mathrm{Ca}^{2+}$ sensitivity of the force generated by muscle fibers under $\beta$-AR stimulation is correlated with this more rapid dissociation of $\mathrm{Ca}^{2+}$ from cTnC. Therefore, increased dissociation of $\mathrm{Ca}^{2+}$ from cTnC, coupled with a more rapid uptake of $\mathrm{Ca}^{2+}$ by the sarcoplasmic reticulum stimulated by PKA phosphorylation of PLB, may account for the more rapid relaxation observed during the inotropic response of the heart to ISO. In the present study, we observed no difference in the $\mathrm{Ca}^{2+}$ sensitivity of myofibrils between the control and tail-suspended rat hearts, but the $\mathrm{Ca}^{2+}$ sensitivity of myofibrils exhibited less reduction following PKA treatment in tail-suspended hearts. Therefore, the working hearts and cardiomyocytes demonstrated a slow relaxation and blunted responsiveness to ISO in 4-week tail-suspended rats.

In summary, $\mathrm{N}$-terminal degradation of $\mathrm{cTnI}$ in tailsuspended rat hearts is a major component to reduce cardiac function responsiveness to ISO in the $\beta$-AR signal transduction pathway.

\section{Acknowledgements}

This study was supported by the National Natural Science Foundation of China (no. 31071044).

\section{References}

1. Layland J, Solaro RJ and Shah AM: Regulation of cardiac contractile function by troponin I phosphorylation. Cardiovase Res 66: 12-21, 2005.

2. Marston SB and Redwood CS: Modulation of thin filament activation by breakdown or isoform switching of thin filament proteins: physiological and pathological implications. Circ Res 93: 1170-1178, 2003.

3. Solaro RJ, Rosevear P and Kobayashi T: The unique functions of cardiac troponin I in the control of cardiac muscle contraction and relaxation. Biochem Biophys Res Commun 369: 82-87, 2008.

4. Fentzke RC, Buck SH, Patel JR, Lin H, Wolska BM, Stojanovic MO, Martin AF, Solaro RJ, Moss RL and Leiden JM: Impaired cardiomyocyte relaxation and diastolic function in transgenic mice expressing slow skeletal troponin I in the heart. J Physiol 517: 143-157, 1999.

5. Arteaga GM, Warren CM, Milutinovic S, Martin AF and Solaro RJ: Specific enhancement of sarcomeric response to $\mathrm{Ca}^{2+}$ protects murine myocardium against ischemia-reperfusion dysfunction. Am J Physiol Heart Circ Physiol 289: H2183-H2192, 2005.

6. Barbato JC, Huang QQ, Hossain MM, Bond M and Jin JP: Proteolytic N-terminal truncation of cardiac troponin I enhances ventricular diastolic function. J Biol Chem 280: 6602-6609, 2005.

7. Takimoto E, Soergel DG, Janssen PM, Stull LB, Kass DA and Murphy AM: Frequency- and afterload-dependent cardiac modulation in vivo by troponin I with constitutively active protein kinase A phosphorylation sites. Circ Res 94: 496-504, 2004.

8. Dong WJ, An J, Xing J and Cheung HC: Structural transition of the inhibitory region of troponin I within the regulated cardiac thin filament. Arch Biochem Biophys 456: 135-142, 2006.

9. Sadayappan S, Finley N, Howarth JW, Osinska H, Klevitsky R, Lorenz JN, Rosevear PR and Robbins J: Role of the acidic $\mathrm{N}^{\prime}$ region of cardiac troponin I in regulating myocardial function. FASEB J 22: 1246-1257, 2008.

10. Yu ZB, Zhang LF and Jin JP: A proteolytic $\mathrm{NH}_{2}$-terminal truncation of cardiac troponin I that is up-regulated in simulated microgravity. J Biol Chem 276: 15753-15760, 2001.

11. Zhang L, Wang YY and Yu ZB: Depressed responsiveness of cardiomyocytes to isoproterenol in simulated weightlessness rats. Sheng Li Xue Bao 59: 845-850, 2007 (In Chinese).

12. Yin W, Liu JC, Fan R, Sun XQ, Ma J, Feng N, Zhang QY, Yin Z, Zhang SM, Guo HT, Bi H, Wang YM, Sun X, Cheng L, Cui Q, Yu SQ, Yi DH and Pei JM: Modulation of $\{$ beta $\}$-adrenoceptor signaling in the hearts of 4-wk simulated weightlessness rats. J Appl Physiol 105: 569-574, 2008. 
13. Morey-Holton ER and Globus RK: Hindlimb unloading rodent model: technical aspects. J Appl Physiol 92: 1367-1377, 2002.

14. Nagata K, Liao R, Eberli FR, Satoh N, Chevalier B, Apstein CS, and Suter TM: Early changes in excitation-contraction coupling: transition from compensated hypertrophy to failure in Dahl saltsensitive rat myocytes. Cardiovasc Res 37: 467-477, 1998.

15. Fabiato A and Fabiato F: Effects of magnesium on contractile activation of skinned cardiac cells. J Physiol 249: 497-517, 1975.

16. MacDonnell SM, García-Rivas G, Scherman JA, Kubo H Chen X, Valdivia H and Houser SR: Adrenergic regulation of cardiac contractility does not involve phosphorylation of the cardiac ryanodine receptor at serine 2808. Circ Res 102: e65-e72, 2008.

17. Stelzer JE, Patel JR, Walker JW and Moss RL: Differential roles of cardiac myosin-binding protein $\mathrm{C}$ and cardiac troponin $\mathrm{I}$ in the myofibrillar force responses to protein kinase A phosphorylation. Circ Res 101: 503-511, 2007.
18. Sadayappan S, Gulick J, Klevitsky R, Lorenz JN, Sargent M, Molkentin JD and Robbins J: Cardiac myosin binding protein-C phosphorylation in a $\{$ beta $\}$-myosin heavy chain background. Circulation 119: 1253-1262, 2009.

19. Sadayappan S, Osinska H, Klevitsky R, Lorenz JN, Sargent M, Molkentin JD, Seidman CE, Seidman JG and Robbins J: Cardiac myosin binding protein $\mathrm{C}$ phosphorylation is cardioprotective. Proc Natl Acad Sci USA 103: 16918-16923, 2006.

20. Li L, Desantiago J, Chu G, Kranias EG and Bers DM: Phosphorylation of phospholamban and troponin I in betaadrenergic-induced acceleration of cardiac relaxation. Am J Physiol Heart Circ Physiol 278: H769-H779, 2000.

21. Saucerman JJ and McCulloch AD: Mechanistic systems models of cell signaling networks: a case study of myocyte adrenergic regulation. Prog Biophys Mol Biol 85: 261-278, 2004.

22. Zhang R, Zhao J, Mandveno A and Potter JD: Cardiac troponin I phosphorylation increases the rate of cardiac muscle relaxation. Circ Res 76: 1028-1035, 1995. 